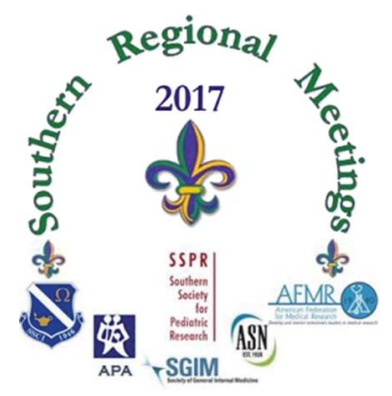

\title{
2017 Southern Regional Meeting
}

\section{AWARD RECIPIENTS}

\author{
Cardiovascular Case Report Award
}

Zachary Bloomer, Dwight David Eisenhower Army Medical Center

Krisitn Lynch, LSU Health Sciences Center

Pardeep Masuta, SUNY Upstate Medical University

Ghulam Murtaza, East Tennessee State University

Peyman Naji, SUNY Upstate Medical University

Nethuja Salagundla, Texas Tech University Health Science Center Amarillo

Karan Seegobin, University of Florida, College of Medicine Jacksonville

Ismael Valle Irizarry, San Juan City Hospital, Auxilio Mutuo Hospital

\section{SAFMR/SSCI Junior Faculty Research Travel Award}

Mohammed Al-Obaide, Texas Tech University Health Science Center

David Green, Emory University

Michael Hall, University of Mississippi Medical Center

Shigeki Saito, Tulane University

Bashar Staitieh, Emory University

Xin Zhang, Ochsner Medical Center

SAFMR/SSCI Trainee Research Award

Efstathia Andrikopoulou, University of Alabama Medical Center

Isabel Anzola, Emory University

Stephen Arndt, Ochsner Clinic Foundation

Adam Bierzynski, Lenox Hill Hospital

Saumeth Cardona, Emory University

Ellis Christian, University of Tennessee Health Science Center

Georgia Davis, Emory University

David Flatt, University of Tennessee Health Science Center

Douglas Friedman, Lenox Hill Hospital

Joseph Gabriel, East Tennessee State University/Quillen College of Medicine

Rohan Goswami, University of Tennessee Health Science Center

Yenal Harper, University of Tennessee Health Science Center

Mark Heckle, University of Tennessee Health Science Center

Alfonso Hernandez-Romieu, Emory University

Diego Hidalgo, East Tennessee State University

Joseph Holthoff, University of Arkansas for Medical Sciences

Mohammad Islam, Texas Tech University Health Science Center Amarillo

Evan Joye, Lenox Hill Hospital

Faisal Kama, University of Tennessee

Michael Levinson, University of Alabama at Birmingham

Nicholas Maurice, Emory University

Asadullah Mirza, Texas Tech University Health Science Center Amarillo 
Sharareh Moraveji, Texas Tech University Health Sciences Center/Paul L. Foster School of Medicine

Lindsay Muncy, Dwight D. Eisenhower Army Medical Center

Nicolas Mungo, East Tennessee State University

Carlos Munoz, Hospital Municipal de San Juan

Laura Petrauskas, LSU Health Sciences Center

Ahmed Qasim, Texas Tech University Health Science Center Amarillo

Beatrix Ramos, University of Tennessee Health Science Center

Derek Russell, University of Alabama at Birmingham

Maria Salguero Bermonth, Texas Tech University Health Science Center Amarillo

Corey Saraceni, LSU Health Sciences Center

Mary Elizabeth Sexton, Emory University

Lisa Shumate, University of Oklahoma

Katerina Tsegka, Emory University

Milagros Yunes, San Juan City Hospital

Aline Zouk, University of Alabama at Birmingham

SAFMR/SSCI Student Research Award

John Ahn, Medical College of Georgia at Augusta University

Pablo Altieri, University of Puerto Rico, Medical Sciences Campus

Maham Awan, Medical University of South Carolina

Christopher Carr, Tulane University School of Medicine, Tulane University School of Public Health \& Tropical Medicine

Austin Castillo, Texas Tech University Health Sciences Center

Katie Clark, LSU Health Shreveport

Corinne Czerwinski, St .George's University of London

Yang Du, Tulane School of Public Health and Tropical Medicine

Johanna Gerwer, Texas Tech University Health Sciences Center El Paso

Shane Hagen, University of Tennessee Health Science Center

Vikisha Hazariwala, Nova Southeastern University

Sharon Hsieh, Emory University, Atlanta VA Medical Center

Christopher Ives, University of Alabama School of Medicine

John Jasper, University of Tennessee Health Science Center

Jennifer Jo, Emory University

Hilary Keller, UQ-Ochsner School of Medicine, Ochsner Clinic Foundation

Nicole Lee, Marshall University School of Medicine

Anthony Naquin, LSUHSC New Orleans

Tung Nguyen, Texas Tech University Health Science Center Amarillo

Krishna Pandya, Tulane University Health Sciences Center

Sara Parker, University of Mississippi Medical Center

Sarah Smith, Ochsner Medical Center

Ryan Sullivan, Ochsner Medical Center

Brittany Tadesse, The University of Tennessee Health Science Center

Barrett Thompson, University of Tennessee Health Science Center

Anezi Uzendu, University of Alabama Birmingham

Michael Winstead, University of Tennessee Health Science Center

John Woller, Emory University School of Medicine

Hyung Sun Yoo, Ochsner Clinic Foundation

\section{SSCI Nephrology Young Investigator Scholar Award}

Mohammed Alzubaidi, Medical University of South Carolina

Faisal Arif, University of Tennessee Health Science Center

Ryan Downey, Emory University School of Medicine

Azeem Mohammed, Augusta University

Marc Saad, Emory University Hospital

Tarek Saleh, University of Tennessee Health Science Center

Renfang Song, Tulane University

Jason White, Augusta University

Santosh Yadav, Tulane University Health Sciences Center 
SSCI Poster Award Finalists

Austin Castillo, Texas Tech University Health Sciences Center

Christina Kearse, Medical University of South Carolina

Lucian Marts, Emory University

SSCI Young Investigator Award

Valeria Cantos, Emory University

Nidhi Jain, University of Tennessee, Memphis

Mohamed Khass, University of Alabama Birmingham

SSCI Young Investigator for General Medicine

Alex Galloway, University of Tennessee Health Science Center College of Medicine

SSPR Basic Science Young Investigator Award Finalists

Emily Masoumy, Children's Hospital of Georgia at Augusta

Michael Sorrell, University of Texas Health Science Center

Aaron Yee, University of Alabama at Birmingham

SSPR Clinical Science Young Investigator Award Finalists

Sarah Coors, Baylor College of Medicine, Texas Children's Hospital

Ariel Salas, University of Alabama at Birmingham

Colm Travers, University of Alabama at Birmingham

SSPR David Oelberg Honorary Trainee Travel Award

Aarthi Gunasekaran, Oklahoma University Health Sciences Center

SSPR Young Faculty Travel Award

Hala Chaaban, Oklahoma University Health Sciences Center

Netsanet Kassa, Oklahoma University Health Sciences Center

Charitharth Lal, University of Alabama at Birmingham

Krithika Lingappan, Baylor College of Medicine

Ravi Patel, Emory University School of Medicine, Children's Healthcare of Atlanta

Brian Stansfield, Augusta University

\section{SSPR Trainee Travel Award}

Chaim Aron, University of Texas at Houston

Vickie Bailey, Oklahoma University Health Sciences Center

Taylor Fitzpatrick-Schmidt, Tulane University, Tulane University School of Medicine

Steven Ford, Texas Children's Hospital

Pankaj Jain, University of Alabama at Birmingham

Brett LaBrecque, University of Alabama at Birmingham

Erica Marxen, University of Oklahoma Health Sciences Center

Rebecca Moore, Medical University of South Carolina

Vershanna Morris, McGovern Medical School University of Texas Health

Radhika Narang, University of Mississippi Medical Center

Candice Nolan, University of Texas Health Science Center Houston-McGovern Medical School

Marcelo Rains, University of Mississippi Medical Center

Sumana Ramarao, University of Mississippi Medical Center

Robert Richter, University of Alabama at Birmingham

Lisa Scheid, UT Southwestern Medical Center

Elizabeth Schulz, Medical University of South Carolina

Lochan Subedi, University of Kentucky

Sunnie Wong, Tulane University School of Medicine 